\title{
Selection of Inbred Lines for Breeding of Maize with High Efficiency in Iron Utilization
}

\author{
Yan Li*, Bingxue Dong*, Chunqing Zhang\# \\ State Key Laboratory of Crop Biology, Shandong Collaborative Innovation Center of High-Yield and \\ High-Efficient Annual Production of Wheat and Corn, Shandong Key Laboratory of Crop Biology, \\ College of Agronomy, Shandong Agricultural University, Taian, China \\ Email: ${ }^{\text {cqzhang@sdau.edu.cn }}$
}

Received 24 March 2014; revised 25 April 2014; accepted 5 May 2014

Copyright (C) 2014 by authors and Scientific Research Publishing Inc.

This work is licensed under the Creative Commons Attribution International License (CC BY). http://creativecommons.org/licenses/by/4.0/

(c) (i) Open Access

\begin{abstract}
Crops are often subjected to iron (Fe)-deficiency due to the limited solubility of this essential element in most neutral or basic soils. Developing cultivars with high efficiency in Fe utilization via breeding programs can provide solutions to this problem as a long term strategy. In the present study, to select inbred lines for breeding of maize with high efficiency in Fe utilization, we screened 123 inbred lines at the seedling stage by analyzing secretion pattern of phytosiderophores, a class of non-protein amino acids released by graminaceous species for Fe utilization, using high-performance liquid chromatography. One hundred and twenty three inbred lines were clustered into nine groups. The low PS secretion rate under Fe-sufficient condition and high PS secretion rate increment after Fe-deficiency treatment type were the ideal inbred lines for breeding of maize with high efficiency in Fe utilization.
\end{abstract}

\section{Keywords}

Maize, Phytosiderophore, Iron-Efficient Utilization

\section{Introduction}

Iron (Fe) is one of the essential nutrients for plant growth, which plays important roles in many crucial metabolic pathways [1]. Although it is the fourth most common element in the Earth's crust, plants are often subjected to Fe-deficiency due to its low solubility especially in calcareous soils (about 30\% of world's cultivated soils) [2] [3]. In world-wide agricultural production, it is an acute contradiction between Fe-abundance in soils and Fe-

\footnotetext{
${ }^{*}$ These authors contributed equally to this paper.

${ }^{\#}$ Corresponding author.
} 
deficiency in crops. Although this issue can be solved through soil improvement and foliage spray of Fe fertilizer, it is a real economic burden. Developing cultivars with high efficiency in Fe utilization via breeding programs can provide solutions to this problem as a long term strategy.

Under Fe-deficiency stress condition, plants have evolved two distinct strategies to solubilize and transport Fe [4]. Strategy I plants, including dicotyledonous and non-graminaceous monocotyledonous species, are characterized by release of proton $\left(\mathrm{H}^{+}\right)$and $\mathrm{Fe}(\mathrm{III})$-chelate reductase to increase Fe-acquisition [5]. Graminaceous species (strategy II plants) secrete phytosiderophores (PSs), a class of non-protein amino acids, into the rhizosphere to solubilize and utilize insoluble Fe (III) in soils [1].

It has been demonstrated that PS secretion rate varies with different graminaceous species, and even with different cultivars within the same species [4] [6]. In addition, Fe-deficiency treatment increases PS secreting rate dramatically [4] [7]. So, it is necessary to characterize PS secreting pattern for developing cultivars of graminaceous species with high efficiency in Fe utilization. At present, no publications on this field are available.

In the present study, to select inbred lines suited to develop maize hybrids with high efficiency in Fe utilization, PS secretion patterns of 123 inbred lines were analyzed. These lines were clustered into nine groups based on PS secretion rates under Fe-sufficient condition and PS secretion rate increment after Fe-deficiency treatment. And then, lines suited to developing cultivars with high efficiency in Fe utilization were discussed. We believe that our work will benefit the Fe-efficient maize breeding.

\section{Materials and Methods}

\subsection{Plant Materials and Growth Condition}

One hundred and twenty three maize inbred lines were used in the present study, which were displayed in Table 1. Seeds were incubated on moist paperbed at $25^{\circ} \mathrm{C}$ for germination. Germinated seeds were transferred to a net floating on a continuously aerated Hoagland's solution ( $\mathrm{pH}$ 6.0) in the temperature-controlled growth chamber

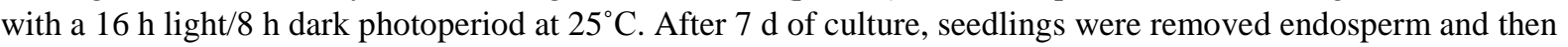
transferred into the aerated Hoagland's solution without Fe element for seven days for collection of root exudates under Fe-deficiency stress. Root exudates under Fe-efficient condition were collected from plants cultured in Hoagland's solution with every essential element. All experiments were performed with three replicates.

\subsection{Collection of Root Exudates}

Firstly, roots of maize seedling were washed clean with deionized water, and then placed in $500 \mathrm{ml}$ of deionized water for collection of root exudates. Root exudates collection was started at 7:00 and terminated at 15:00, lasting eight hours.

\subsection{High-Performance Liquid Chromatography (HPLC) Analysis of PS}

Root exudates were chromatographed on Amberlite IR120B ( $\mathrm{H}^{+}$form) followed by freeze-dried in vacuum. The freeze-dried materials were dissolved in $10 \mathrm{ml}$ ultrapure water, and then analyzed by HPLC using C18 column $(4.6 \mathrm{~mm} \times 250 \mathrm{~mm})$ with wavelength $209 \mathrm{~nm}$, mobile phase $0.5 \%\left(\mathrm{NH}_{4}\right) \mathrm{H}_{2} \mathrm{PO}_{4}(\mathrm{pH} 2.85)$ and flow rate 0.6 $\mathrm{ml} \cdot \mathrm{min}^{-1}$.

\subsection{Statistical Analysis}

DPS 7.05 software was used for data processing [8]. Chi-square distance and sum of squared deviations were adopted in cluster analysis.

\section{Results}

\subsection{PS Secretion Characteristics of the 123 Inbred Lines at Seedling Stage}

HPLC analysis was performed to characterize PS secretion of 123 inbred lines at seedling stage. Table 1 listed the PS secretion data of these lines. The average of PS secretion rate under Fe-sufficient condition was 7.281 $\mathrm{mg} /(\mathrm{g}$ dry root*8h). The variation range among different genotypes was from $1.786 \pm 0.222 \mathrm{mg} /(\mathrm{g}$ dry root*8h) [mean \pm standard deviation (S.D.), Dan340] to $19.695 \pm 0.442 \mathrm{mg} /(\mathrm{g}$ dry root*8h) (LL0726-1). The average of 
Table 1. Phytosiderophores (PS) secretion rate $\left[\mathrm{mg} \cdot \mathrm{g}^{-1}\right.$ dry root $(8 \mathrm{~h})^{-1}$ ] of 123 maize inbred lines under Fe-sufficient condition $(+\mathrm{Fe})$ and after Fe-deficiency.

\begin{tabular}{|c|c|c|c|c|c|}
\hline Inbred lines & $\begin{array}{l}\text { PS secretion rate }(+\mathrm{Fe}) \\
\text { [mg/g dry root*8h] }\end{array}$ & $\begin{array}{l}\text { PS secretion rate }(-\mathrm{Fe}) \\
{[\mathrm{mg} / \mathrm{g} \text { dry root } * 8 \mathrm{~h}]}\end{array}$ & Inbred lines & $\begin{array}{l}\text { PS secretion rate }(+\mathrm{Fe}) \\
\text { [mg/g dry root*8h] }\end{array}$ & $\begin{array}{l}\text { PS secretion rate }(-\mathrm{Fe}) \\
{[\mathrm{mg} / \mathrm{g} \text { dry root*8h] }}\end{array}$ \\
\hline 196 & $4.754 \pm 0.252$ & $4.958 \pm 0.217$ & 04qun0631-1 & $5.474 \pm 0.376$ & $7.386 \pm 0.276$ \\
\hline 502 & $7.196 \pm 0.068$ & $7.653 \pm 0.414$ & 04qun-12-2 & $6.856 \pm 0.818$ & $11.427 \pm 0.767$ \\
\hline 543 & $6.296 \pm 0.251$ & $8.084 \pm 0.409$ & 04qun-22-1 & $5.396 \pm 0.513$ & $6.134 \pm 0.362$ \\
\hline 933 & $10.084 \pm 0.538$ & $13.796 \pm 1.377$ & 04qun-33 & $3.903 \pm 0.205$ & $11.942 \pm 0.927$ \\
\hline 1029 & $4.515 \pm 0.118$ & $7.670 \pm 0.991$ & $958-1-9$ & $6.615 \pm 0.226$ & $9.112 \pm 1.744$ \\
\hline 2102 & $13.170 \pm 0.528$ & $23.335 \pm 0.798$ & 99qun-8-2 & $4.404 \pm 0.112$ & $4.446 \pm 0.089$ \\
\hline 3189 & $9.753 \pm 0.192$ & $10.524 \pm 0.530$ & A150-3-1 & $2.626 \pm 0.232$ & $5.330 \pm 0.282$ \\
\hline 3841 & $9.393 \pm 0.333$ & $10.151 \pm 0.908$ & A210-1 & $10.583 \pm 1.027$ & $12.706 \pm 0.486$ \\
\hline 3904 & $10.826 \pm 0.339$ & $11.017 \pm 0.883$ & A22 & $5.536 \pm 0.484$ & $6.234 \pm 0.408$ \\
\hline 4866 & $4.768 \pm 0.318$ & $6.684 \pm 0.263$ & A348-4-2 & $6.389 \pm 0.031$ & $6.692 \pm 0.141$ \\
\hline 5005 & $5.079 \pm 0.628$ & $7.685 \pm 0.765$ & A369-2-1 & $3.432 \pm 0.370$ & $5.989 \pm 0.362$ \\
\hline 5237 & $13.143 \pm 0.169$ & $14.023 \pm 0.186$ & A6-2-1 & $11.629 \pm 1.571$ & $13.734 \pm 0.688$ \\
\hline 8723 & $3.474 \pm 0.179$ & $3.969 \pm 0.073$ & B jian8 & $5.552 \pm 0.618$ & $8.775 \pm 0.167$ \\
\hline 9418 & $5.173 \pm 0.329$ & $14.945 \pm 0.037$ & B104-1-2 & $9.297 \pm 0.200$ & $13.021 \pm 0.256$ \\
\hline 52106 & $5.134 \pm 0.346$ & $7.799 \pm 0.451$ & B117 & $3.566 \pm 0.581$ & $7.103 \pm 0.134$ \\
\hline $4880-1-4$ & $4.845 \pm 0.445$ & $5.320 \pm 0.213$ & $\mathrm{~B} 121$ & $4.361 \pm 0.276$ & $8.766 \pm 1.252$ \\
\hline 03qun-3-3 & $10.311 \pm 0.524$ & $12.291 \pm 0.833$ & B138-1 & $9.418 \pm 0.568$ & $12.354 \pm 1.032$ \\
\hline 03qun-8-2 & $7.156 \pm 0.129$ & $7.491 \pm 0.154$ & B178-1 & $5.782 \pm 0.374$ & $7.506 \pm 0.544$ \\
\hline 04qun0601-3 & $5.034 \pm 0.294$ & $5.114 \pm 0.318$ & B209 & $4.108 \pm 0.347$ & $4.165 \pm 0.456$ \\
\hline 04qun0603-2 & $8.072 \pm 0.558$ & $10.320 \pm 0.599$ & B217-2-2 & $18.275 \pm 1.066$ & $19.069 \pm 0.408$ \\
\hline 04qun0603-9 & $7.533 \pm 0.272$ & $8.554 \pm 0.446$ & B223-1 & $6.523 \pm 0.727$ & $11.162 \pm 1.419$ \\
\hline 04qun0604-4 & $6.218 \pm 0.774$ & $8.379 \pm 0.203$ & B280-1-1 & $8.134 \pm 0.238$ & $8.771 \pm 0.463$ \\
\hline 04qun0611-3 & $8.329 \pm 0.508$ & $11.877 \pm 0.273$ & B283 & $19.124 \pm 0.203$ & $19.414 \pm 0.951$ \\
\hline B302 & $7.109 \pm 0.442$ & $7.641 \pm 0.507$ & HN0709-1-1 & $13.109 \pm 0.580$ & $24.526 \pm 1.518$ \\
\hline B38-1 & $10.649 \pm 0.545$ & $17.866 \pm 0.453$ & HN0713-2-1 & $4.195 \pm 0.592$ & $6.427 \pm 0.450$ \\
\hline B40-2 & $6.113 \pm 0.184$ & $6.200 \pm 0.061$ & HN0718-2-1 & $7.357 \pm 0.230$ & $9.440 \pm 0.464$ \\
\hline B50 & $12.111 \pm 1.158$ & $15.440 \pm 1.387$ & Huang C & $9.643 \pm 0.548$ & $10.080 \pm 0.309$ \\
\hline B52 & $3.359 \pm 0.157$ & $5.759 \pm 0.317$ & Huangyesi & $5.242 \pm 0.085$ & $5.349 \pm 0.223$ \\
\hline B57 & $5.225 \pm 0.422$ & $9.400 \pm 0.686$ & Huangzaosi & $6.578 \pm 0.600$ & $7.012 \pm 0.271$ \\
\hline Cai 11-8 & $5.722 \pm 0.395$ & $6.834 \pm 0.726$ & Ji 842 & $4.668 \pm 0.574$ & $7.053 \pm 0.419$ \\
\hline Chang 7-2 & $5.750 \pm 0.627$ & $6.765 \pm 0.792$ & Ji 853 & $4.751 \pm 0.749$ & $5.816 \pm 0.731$ \\
\hline D10-2 & $2.377 \pm 0.257$ & $2.414 \pm 0.200$ & $\mathrm{~K} 12$ & $5.141 \pm 0.416$ & $6.337 \pm 0.296$ \\
\hline D18-1-1 & $6.743 \pm 0.699$ & $7.889 \pm 0.696$ & LL0706-1-1 & $14.680 \pm 0.403$ & $15.473 \pm 0.161$ \\
\hline D25-2-1 & $7.400 \pm 0.387$ & $7.953 \pm 0.118$ & LL0726-1 & $19.695 \pm 0.442$ & $22.915 \pm 1.277$ \\
\hline D26-2 & $7.198 \pm 0.216$ & $7.525 \pm 0.251$ & Longkang 11 & $9.781 \pm 0.433$ & $12.727 \pm 0.743$ \\
\hline Dan 340 & $1.786 \pm 0.222$ & $3.684 \pm 0.783$ & Lp08-17 & $6.992 \pm 0.127$ & $7.879 \pm 0.290$ \\
\hline Danhuang 02 & $4.335 \pm 0.234$ & $5.138 \pm 0.371$ & Lp08-19 & $5.217 \pm 0.596$ & $7.149 \pm 0.958$ \\
\hline Danhuang 212 & $3.191 \pm 0.205$ & $7.858 \pm 0.411$ & M54 & $3.223 \pm 0.891$ & $7.341 \pm 0.852$ \\
\hline Dasui-1-3 & $3.786 \pm 0.438$ & $9.071 \pm 0.550$ & NH07001-3-1 & $6.017 \pm 0.652$ & $11.029 \pm 0.232$ \\
\hline E2-1-1 & $6.426 \pm 0.376$ & $6.920 \pm 0.492$ & $\mathrm{P} 138$ & $3.451 \pm 0.653$ & $4.710 \pm 0.083$ \\
\hline E3-1-2 & $7.915 \pm 0.505$ & $8.541 \pm 0.704$ & $\mathrm{PH} 4 \mathrm{CV}$ & $12.159 \pm 0.541$ & $14.875 \pm 0.572$ \\
\hline
\end{tabular}




\begin{tabular}{|c|c|c|c|c|c|}
\hline \multicolumn{6}{|l|}{ Continued } \\
\hline E4-2 & $3.357 \pm 0.622$ & $4.773 \pm 0.900$ & Pr07001-1 & $12.234 \pm 0.043$ & $14.600 \pm 1.625$ \\
\hline F22-1 & $5.571 \pm 0.279$ & $5.641 \pm 0.056$ & Pr07142 & $8.890 \pm 1.295$ & $11.431 \pm 0.515$ \\
\hline Fangxi & $7.833 \pm 0.584$ & $8.495 \pm 0.161$ & pr07148 & $12.176 \pm 0.932$ & $12.758 \pm 1.550$ \\
\hline H21 & $4.967 \pm 0.574$ & $10.059 \pm 0.790$ & Pr07169 & $11.974 \pm 0.743$ & $14.400 \pm 0.790$ \\
\hline HB08F28-1 & $8.801 \pm 0.235$ & $10.491 \pm 0.882$ & Pr07357 & $2.937 \pm 0.111$ & $9.835 \pm 0.147$ \\
\hline HB08II-37 & $14.343 \pm 0.092$ & $16.086 \pm 0.798$ & Pr07483 & $3.397 \pm 0.382$ & $14.380 \pm 0.971$ \\
\hline HN0701-1-1 & $9.902 \pm 0.424$ & $10.722 \pm 0.264$ & Pr07504 & $2.531 \pm 0.241$ & $9.524 \pm 1.562$ \\
\hline Pr7404 & $5.543 \pm 0.464$ & $9.485 \pm 0.748$ & Y36-1 & $9.511 \pm 1.456$ & $10.866 \pm 0.618$ \\
\hline Qi310 & $7.715 \pm 0.646$ & $9.757 \pm 0.604$ & Y4-2 & $10.628 \pm 0.279$ & $15.264 \pm 0.547$ \\
\hline Qichang-3-1 & $10.026 \pm 0.297$ & $10.564 \pm 0.233$ & Y5-2 & $7.849 \pm 0.290$ & $8.487 \pm 0.469$ \\
\hline Ren-4-1 & $13.079 \pm 0.742$ & $17.807 \pm 0.206$ & Y8-1-2 & $10.029 \pm 0.580$ & $14.358 \pm 1.352$ \\
\hline Shen118 & $9.854 \pm 0.465$ & $12.000 \pm 0.093$ & Yang3-2-1 & $6.055 \pm 0.279$ & $9.534 \pm 0.522$ \\
\hline Shen137 & $6.759 \pm 0.077$ & $14.061 \pm 1.500$ & Ye 478 & $4.104 \pm 0.119$ & $6.659 \pm 0.635$ \\
\hline Sizisi & $4.195 \pm 0.563$ & $4.957 \pm 0.185$ & Ye 488 & $2.702 \pm 0.156$ & $3.010 \pm 0.197$ \\
\hline VT187 & $2.241 \pm 0.982$ & $2.423 \pm 0.383$ & Ye 8001 & $5.015 \pm 0.371$ & $5.144 \pm 0.190$ \\
\hline W618 & $4.411 \pm 0.356$ & $5.579 \pm 0.968$ & ZH-2-1 & $6.033 \pm 0.292$ & $6.294 \pm 0.392$ \\
\hline $\mathrm{x} 178-1$ & $12.431 \pm 0.672$ & $12.684 \pm 0.515$ & 05ZH-3-1 & $13.831 \pm 0.746$ & $14.819 \pm 0.779$ \\
\hline Y17-2 & $3.869 \pm 0.449$ & $4.074 \pm 0.368$ & Zheng 58 & $5.914 \pm 0.301$ & $7.634 \pm 0.798$ \\
\hline Y3-2 & $5.083 \pm 0.258$ & $5.794 \pm 1.203$ & Zheng 653 & $7.471 \pm 0.481$ & $9.914 \pm 0.511$ \\
\hline Y32-1-1 & $7.031 \pm 1.057$ & $9.928 \pm 0.366$ & 7bonghung 204 & $7204+0126$ & 217 \\
\hline Y30-1-1 & $11.755 \pm 1.057$ & $12.510 \pm 0.481$ & Znongnuang <U4 & $7.204 \pm 0.120$ & $10.104 \pm 0.342$ \\
\hline
\end{tabular}

PS secretion rate after Fe-deficiency stress was $9.560 \mathrm{mg} /(\mathrm{g}$ dry root*8h), and the variation range was from $2.414 \pm 0.200 \mathrm{mg} /(\mathrm{g}$ dry root*8h) (D10-2) to $24.526 \pm 1.518 \mathrm{mg} /(\mathrm{g}$ dry root*8h) (HN0709-1-1). These data strongly suggested that PS secrete rates were clearly different among these inbred lines at the seedling stage under Fe-sufficient condition and under Fe-deficiency treatment. In addition, Fe-deficiency treatment increased PS secretion rate among all 123 inbred lines. And the increment was from $0.037 \mathrm{mg} /(\mathrm{g}$ dry root*8h) (D10-2) to $11.417 \mathrm{mg} /(\mathrm{g}$ dry root*8h) (HN0709-1-1). These data revealed that sensibility to Fe-deficiency treatment was obviously different among these inbred lines.

\subsection{Clustering Analysis of the 123 Inbred Lines Based on PS Secretion Characteristics at the Seedling Stage}

Figure 1 listed the data gained from Chi-square distance and sum of squared deviations analysis of the 123 inbred lines. These lines were grouped based on their PS secretion rates under Fe-sufficient condition (Figure 1(a)) and PS secretion rate increment after Fe-deficiency treatment (Figure 1(b)).

With the threshold value 15.20, these inbred lines can be categorized into three groups based on the PS secretion rate under Fe-sufficient condition: low $(\mathrm{L})$, intermediate $(\mathrm{M})$ and high $(\mathrm{H})$ (Figure 1(a)). The average secretion rates of the three groups were 4.307, 7.001 and $11.792 \mathrm{mg} /(\mathrm{g}$ dry root*8h) respectively, raging from 1.786 to $5.782 \mathrm{mg} /(\mathrm{g}$ dry root*8h), from 5.914 to $8.329 \mathrm{mg} /(\mathrm{g}$ dry root*8h), and from 5.914 to $19.695 \mathrm{mg} /(\mathrm{g}$ dry root $8 \mathrm{~h}$ ) respectively. With the threshold value 12.82 , these lines were also categorized into three groups based on PS secretion rate increment after Fe-deficiency treatment: low (l), intermediate (m) and high (h) (Figure 1(b)). The average secretion rate increment of the three groups were $0.595,2.382$ and $5.792 \mathrm{mg} /(\mathrm{g}$ dry root*8h) respectively, raging from 0.037 to $1.416 \mathrm{mg} /(\mathrm{g}$ dry root*8h), from 1.690 to $3.329 \mathrm{mg} /(\mathrm{g}$ dry root*8h), and from 3.479 to $11.417 \mathrm{mg} /(\mathrm{g}$ dry root*8h) respectively.

To cluster the 123 inbred lines more accurately, these lines were divided into nine groups based on both the two parameters described above (Table 2). The nine groups were: 1) the high PS secretion rate under Fe-sufficient condition and high PS secretion rate increment after Fe-deficiency treatment (Hh); 2) the high PS secretion rate and intermediate PS secretion rate increment $(\mathrm{Hm})$; 3) the high PS secretion rate and low PS secretion rate 


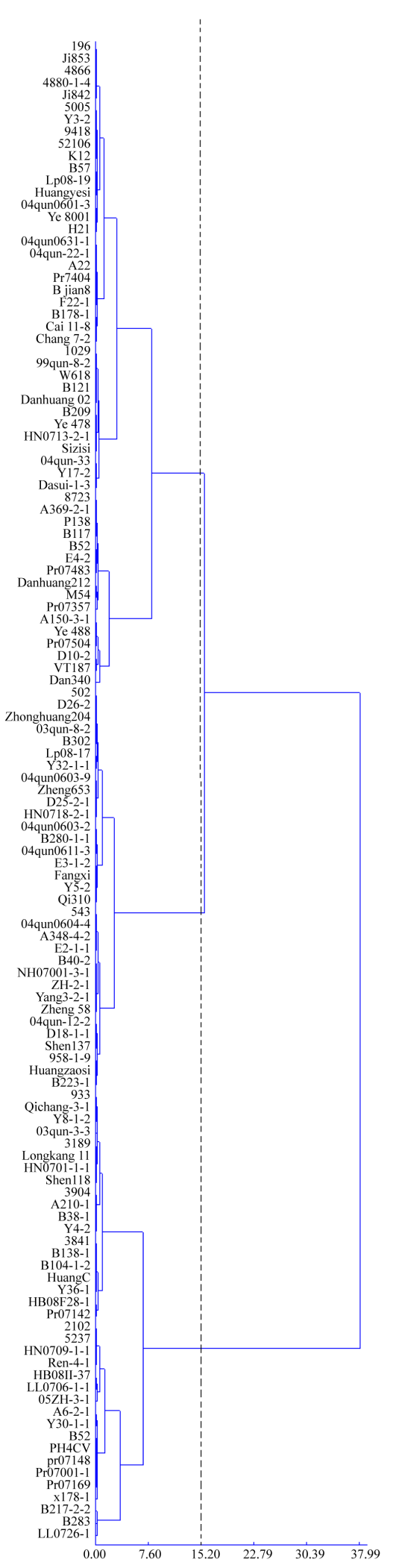

(a)

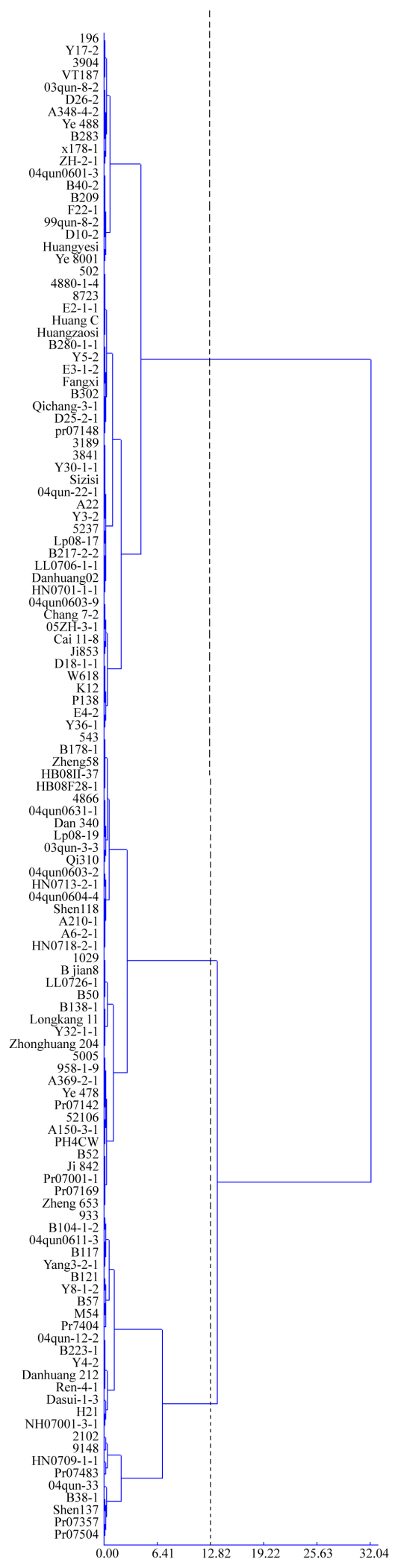

(b)

Figure 1. Clustering of 123 maize inbred lines based on PS secretion rate under Fe-sufficient condition (a) and PS secretion rate increment after Fe-deficiency treatment (b). 
Table 2. Grouping of 123 maize inbred lines based on PS secretion rate $\left[\mathrm{mg} \cdot \mathrm{g}^{-1}\right.$ dry root $\left.(8 \mathrm{~h})^{-1}\right]$ under Fe-sufficient condition $(+\mathrm{Fe})$ and PS secretion rate increment after Fe-deficiency treatment $(-\mathrm{Fe})$.

\begin{tabular}{|c|c|c|c|}
\hline$+\mathrm{Fe}-\mathrm{Fe}$ & $\begin{array}{c}\text { high PS secretion rate } \\
\text { increment [h, } 3.479-11.417 \\
\mathrm{mg} /(\mathrm{g} \text { dry root*8h)] }\end{array}$ & $\begin{array}{c}\text { intermediate PS secretion rate increment } \\
{[\mathrm{m}, 1.690-3.329 \mathrm{mg} /(\mathrm{g} \text { dry root*8h)] }}\end{array}$ & $\begin{array}{c}\text { low PS secretion rate increment } \\
{[1,0.037-1.416 \mathrm{mg} /(\mathrm{g} \text { dry root*8h) }}\end{array}$ \\
\hline $\begin{array}{c}\text { High PS secretion rate } \\
\text { [H, } 8.801-19.695 \\
\text { mg/(g dry root*8h)] }\end{array}$ & $\begin{array}{l}\text { 933, 2102, B104-1-2, } \\
\text { B38-1, HN0709-1-1, } \\
\text { Ren-4-1, Y4-2, Y8-1-2 }\end{array}$ & $\begin{array}{l}\text { 03qun-3-3, A210-1, A6-2-1, } \\
\text { B138-1, B50, HB08F28-1, } \\
\text { HB08II-37, LL0726-1, } \\
\text { Longkang 11, PH4CV, } \\
\text { Pr07001-1, Pr07142, Pr07169, } \\
\text { Shen118 }\end{array}$ & $\begin{array}{l}\text { 3189, 3841, 3904, 5237, } \\
\text { B217-2-2, B283, HN0701-1-1, } \\
\text { Huang C, LL0706-1-1, } \\
\text { pr07148, Qichang-3-1, x178-1, } \\
\text { Y30-1-1, Y36-1, 05ZH-3-1 }\end{array}$ \\
\hline $\begin{array}{c}\text { Intermediate PS } \\
\text { secretion rate [M, } \\
5.914-8.329 \\
\text { mg/(g dry root*8h)] }\end{array}$ & $\begin{array}{l}\text { 04qun0611-3, 04qun-12-2, } \\
\text { B223-1, NH07001-3-1, } \\
\text { Shen137, Yang 3-2-1 }\end{array}$ & $\begin{array}{l}\text { 543, 04qun0603-2, } \\
\text { 04qun0604-4, 958-1-9, } \\
\text { HN0718-2-1, Qi310, Y32-1-1, } \\
\text { Zheng 58, Zheng 653, } \\
\text { Zhonghuang } 204\end{array}$ & $\begin{array}{l}\text { 502, 03qun-8-2, 04qun0603-9, } \\
\text { A348-4-2, B280-1-1, B302, } \\
\text { B40-2, D18-1-1, D25-2-1, } \\
\text { D26-2, E2-1-1, E3-1-2, Fangxi, } \\
\text { Huangzaosi, Lp08-17, Y5-2, } \\
\text { ZH-2-1 }\end{array}$ \\
\hline $\begin{array}{c}\text { low PS secretion rate } \\
\text { [L, } 1.786-5.782 \\
\mathrm{mg} /(\mathrm{g} \text { dry root*8h)] }\end{array}$ & $\begin{array}{l}\text { 9418, 04qun-33, B117, } \\
\text { B121, B57, Danhuang 212, } \\
\text { Dasui-1-3, H21, M54, } \\
\text { Pr07357, Pr07483, Pr07504, } \\
\text { Pr7404 }\end{array}$ & $\begin{array}{l}\text { 1029, 4866, 5005, 52106, } \\
\text { 04qun0631-1, A150-3-1, } \\
\text { A369-2-1, B jian8, B178-1, } \\
\text { B52, Dan 340, HN0713-2-1, } \\
\text { Ji 842, Lp08-19, Ye } 478\end{array}$ & $\begin{array}{l}\text { 196, 8723, 4880-1-4, } \\
\text { 04qun0601-3, 04qun-22-1, } \\
\text { 99qun-8-2, A22, B209, } \\
\text { Cai 11-8, Chang 7-2, D10-2, } \\
\text { Danhuang 02, E4-2, F22-1, } \\
\text { Huangyesi, Ji 853, K12, P138, } \\
\text { Sizisi, VT187, W618, Y17-2, } \\
\text { Y3-2, Ye 488, Ye 8001 }\end{array}$ \\
\hline
\end{tabular}

increment (Hl); 4) the intermediate PSs secretion rate and high PSs secretion rate increment (Mh); 5) the intermediate PS secretion rate and intermediate PS secretion rate increment (Mm); 6) the intermediate PS secretion rate and low PS secretion rate increment (Ml); 7) the low PS secretion rate and high PS secretion rate increment (Lh); 8) the low PS secretion rate and intermediate PS secretion rate increment (Lm); 9) the low PS secretion rate and low PS secretion rate increment (Ll) (Table 2).

\section{Discussion}

Fe is one of the essential nutrients for microorganisms, plants, animals and human beings. Fe in plants is an important source of this element for human beings, especially in developing countries [9]. However, plants are often subjected to Fe-deficiency due to the limited solubility of Fe in most neutral or basic soils [3]. In graminaceous species, PSs are the ones which are secreted into the rhizosphere to solubilize and utilize insoluble Fe (III) [5]. In the present study, to select inbred lines suited to developing maize hybrids with high efficiency in Fe utilization, we characterize PS secretion pattern of 123 lines.

In this study, PS secretion rate ranged from $1.786 \pm 0.222$ to $19.695 \pm 0.442 \mathrm{mg} /(\mathrm{g}$ dry root*8h) under Fesufficient condition, and PS secretion rate increment ranged from 0.037 to $11.417 \mathrm{mg} /(\mathrm{g}$ dry root*8h) under Fedeficiency stress, suggesting that PS secretion pattern differed with genotypes.

Based on PS secretion rates under Fe-sufficient condition and PS secretion rate increment after Fe-deficiency treatment, 123 maize inbred lines were divided into nine groups, $\mathrm{Hh}, \mathrm{Hm}, \mathrm{Hl}, \mathrm{Mh}, \mathrm{Mm}, \mathrm{Ml}, \mathrm{Lh}, \mathrm{Lm}$ and $\mathrm{Ll}$ (Table 2). PSs are metal chelators that play a major role in Fe and Zn acquisition [10] [11]. So it is unnecessary for plants cultured in Hoagland's solution to secrete large amounts of PSs. Under Fe-deficient stress, however, more increase of PS secretion rate contributes evidently to quickly adapt the stress circumstance. Therefore, the Lh type was the ideal inbred lines for breeding of maize with high efficiency in Fe utilization.

\section{Conclusion}

In the present study, to select inbred lines suited to develop maize hybrids with high efficiency in Fe utilization, PS secretion patterns of 123 inbred lines were analyzed. These lines were clustered into nine groups based on PS secretion rates under Fe-sufficient condition and PS secretion rate increment after Fe-deficiency treatment. The Lh type was the ideal inbred lines for breeding of maize with high efficiency in Fe utilization. 


\section{Acknowledgements}

This work was supported by the National Natural Science Foundation of China (31101160), Seed Improvement Project of Shandong Province, China (2008-6) and Innovative Team Foundation of Modern Agricultural Technology System of Shandong Province, China.

\section{References}

[1] Kobayashi, T. and Nishizawa, N.K. (2012) Iron Uptake, Translocation, and Regulation in Higher Plants. Annual Review of Plant Biology, 63, 131-152. http://dx.doi.org/10.1146/annurev-arplant-042811-105522

[2] Chen, Y. and Barak, P. (1982) Iron Nutrition of Plants in Calcareous Soils. Advances in Agronomy, 35, 217-240.

[3] Hindt, M.N. and Guerinot, M.L. (2012) Getting a Sense for Signals: Regulation of the Plant Iron Deficiency Response. Biochimica Biophysica Acta (BBA)—Molecular Cell Research, 1823, 1521-1530. http://dx.doi.org/10.1016/j.bbamcr.2012.03.010.

[4] Marschner, H. and Römheld, V. (1994) Strategies of Plants for Acquisition of Iron. Plant and Soil, 165, 261-274. http://dx.doi.org/10.1007/BF00008069

[5] Walker, E.L. and Connolly, E.L. (2008) Time to Pump Iron: Iron-Deficiency-Signaling Mechanisms of Higher Plants. Current Opinion in Plant Biology, 11, 530-535. http://dx.doi.org/10.1016/j.pbi.2008.06.013

[6] Römheld, V. and Marschner, H. (1990) Genotypical Differences among Graminaceous Species in Release of Phytosiderophores and Uptake of Iron Phytosiderophores. Plant and Soil, 123, 147-153. http://dx.doi.org/10.1007/BF00011260

[7] Luo, C.L., Shen, Z.G. and Li, X.D. (2008) Root Exudates Increase Metal Accumulation in Mixed Cultures: Implications for Naturally Enhanced Phytoextraction. Water, Air, and Soil Pollution, 193, 147-154. http://dx.doi.org/10.1007/s11270-008-9678-z

[8] Tang, Q.Y. and Feng, M.G. (2007) DPS Data Processing System: Experimental Design, Statistical Analysis and Data Mining. Science Press, Beijing.

[9] Gómez-Galera, S., Rojas, E., Sudhakar, D., Zhu, C., Pelacho, A.M., Capell, T. and Christou, P. (2010) Critical Evaluation of Strategies for Mineral Fortification of Staple Food Crops. Transgenic Research, 19, 165-180. http://dx.doi.org/10.1007/s11248-009-9311-y

[10] Suzuki, M., Takahashi, M., Tsukamoto, T., Watanabe, S., Matsuhashi, S., Yazaki, J., Kishimoto, N., Kikuchi, S., Nakanishi, H., Mori, S. and Nishizawa, N.K. (2006) Biosynthesis and Secretion of Mugineic Acid Family Phytosiderophores in Zinc-Deficient Barley. Plant Journal, 48, 85-97. http://dx.doi.org/10.1111/j.1365-313X.2006.02853.X

[11] Ishimaru, Y., Bashir, K. and Nishizawa, N.K. (2011) Zn Uptake and Translocation in Rice Plants. Rice, 4, 21-27. http://dx.doi.org/10.1007/s12284-011-9061-3 